\section{Effect of food deprivation on differential eyelid conditioning in New Zealand white and American Dutch rabbits*}

\author{
PETER WILLIAM FREY and DEBRA RAVITZ SHELDON \\ Northwestern University, Evanston, Ill. 60201
}

The effect on differential eyelid conditioning of $18 \mathrm{~h}$ of food deprivation was assessed on New Zealand White and American Dutch rabbits. Food deprivation did not increase the rate of acquisition of the conditioned response, nor did it increase general responsivity. Food deprivation did improve discrimination performance. The Dutch rabbits acquired the $C R$ more rapidly than the albino rabbits and showed a greater responsivity in the conditioning situation. The albino rabbits showed superior discrimination performance.

The present study investigated the effects of food deprivation on learning in an aversive Pavlovian conditioning paradigm. Traditional drive concepts (Hull, 1943, Chap. 14) partition the effects of drive manipulations such as food deprivation into a general drive component that is assumed to potentiate all acquired receptor-effector connections and into a specific drive-stimulus component that provides special cues which can be conditioned to responses in the same manner as more observable external stimuli. Hull assumed that generalized drive and habit strength combine in a multiplicative fashion to determine reaction potential. Therefore, food deprivation should potentiate any response that has been conditioned in a testing situation regularly associated with food deprivation, including those responses motivated by aversive events. Thus, in the present situation, food deprivation should enhance eyelid conditioning performance when the animals are regularly trained in a food-deprived state.

A different prediction for the effects of food deprivation in the present study can be derived from a recent proposal by Estes (1969, p. 67-70). According to Estes' hypothesis, drives act as selective stimulus amplifiers, so that food deprivation should enhance the organism's responsivity to stimuli associated with food intake but should have little effect on stimuli associated with aversive events. Thus, for the rabbit eyelid conditioning paradigm, Estes's theory predicts that food deprivation should have little effect on performance.

One source of confounding in this analysis is the possible effect of food

*This investigation was supported by PHS Research Grants MH 17767 and MH 17264. Reprint requests should be sent to Peter W. Frey, Department of Psychology, Northwestern University, Evanston, I11. 60201. deprivation on the perceived aversiveness of electrical shock. The available evidence with rats (Misanin \& Campbell, 1969) suggests, however, that food deprivation thresholds or the amount of activity elicited by inescapable shock. Thus the present study should provide a good opportunity to examine the validity of the alternative predictions offered by Hull's and Estes's theories.

A second major variable analyzed in the present study was the relative performance of two different rabbit strains. In the past, the New Zealand White rabbit has been the only strain used for eyelid conditioning in this country. A recent study by Rubin \& Brown (1969) reports the successful use of the smaller American Dutch rabbit for behavioral investigation. The present investigation provided a direct comparison of the performance of the two strains in a differential eyelid conditioning paradigm.

\section{METHOD}

The Ss were 16 male New Zealand albino rabbits, weighing 4 to 5 lbs each, and 16 male American Dutch rabbits, weighing $2 \frac{1}{2}$ to $3 \mathrm{lbs}$ each. The rabbits were caged individually and had free access to water. After delivery by a local supplier, the Ss were habituated to their new cages until they were eating regularly. Each rabbit was anesthetized with pentobarbital and surgically prepared by embedding two stainless steel bolts into the top of the skull, one behind and to the animal's left of the bregma and one in front, to the animal's right of the bregma. Two lengths of 26-ga suturing wire were attached below and to either side of the right eye, and a rectangular tab of Velcro Fastener was attached to the right eyelid. After recovering from surgery, each animal was habituated in the conditioning chamber without presentation of tones or shock. Eyeblinks were recorded from a potentiometer mounted upon a small does not alter shock detection or aversion
Plexiglas platform that was attached to the bolts implanted in the S's skull.

During conditioning, the $S$ was restrained in a Plexiglas and plywood stock $(13 \times 14 \times 56 \mathrm{~cm})$ that permitted movement of the limbs inside the stock and partial movement of the head, which protruded above the stock. The animal in the stock was placed in an individual sound-attenuated chamber $(35 \times 45 \times 85 \mathrm{~cm})$ that was illuminated by a 7.5-W bulb located behind the $S$. A background white noise was continuously maintained at $60 \mathrm{~dB}$ (SPL) by a Grason-Stadler noise generator (Model 901B) over twin 4-in. speakers located behind the rabbit.

Eyelid movements were recorded graphically with a counterweighted microtorque potentiometer and a Beckman (504D) oscillograph. The US was a $100-\mathrm{msec}$ constant amperage, $3.5-\mathrm{mA}$ electric shock applied through the two implanted electrodes located below and to either side of the right eye. The CSs were 600 and $1,500-\mathrm{Hz}$ tones presented at $80 \mathrm{~dB}, 20 \mathrm{~dB}$ above the background noise as measured at the level of the rabbits' ears. The CS-US interval was $500 \mathrm{msec}$ and the tone CS overlapped and ended simultaneously with the shock. The St tone $(1,500 \mathrm{~Hz})$ was alternated with the $\mathrm{S}$ - tone $(600 \mathrm{~Hz})$. The intertrial interval was $90 \mathrm{sec}$, and the intershock interval, therefore, was $180 \mathrm{sec}$.

The Dutch and albino rabbits were subdivided into deprived and nondeprived groups such that the experimental design was a 2 by 2 factorial, with strain and food deprivation as the two factors. The food-deprived animals were deprived of their standard laboratory rabbit chow for $18 \mathrm{~h}$ each day prior to the start of each conditioning session. The deprivation manipulation was instituted 3 days prior to the first session. Each animal received at least eight daily 1 -h conditioning sessions of $20 \mathrm{~S}+$ and $20 \mathrm{~S}$-trials.

\section{RESULTS}

Response frequency data were analyzed in this study to determine relative rates of acquisition of the conditioned response and asymptotic levels of discrimination performance. A CR was defined as an eyelid closure during the CS-US interval that produced a $2-\mathrm{mm}$ or greater deflection in the recording pen. The URs elicited by the shock US produced pen deflections of approximately $20 \mathrm{~mm}$ in amplitude. In addition to the calculation of significance level, the present report also estimates the strength of experimental effects by computing $\omega^{2}$ (Vaughan \& Corballis, 1969).

Data analysis did not indicate any interactions between the two main 


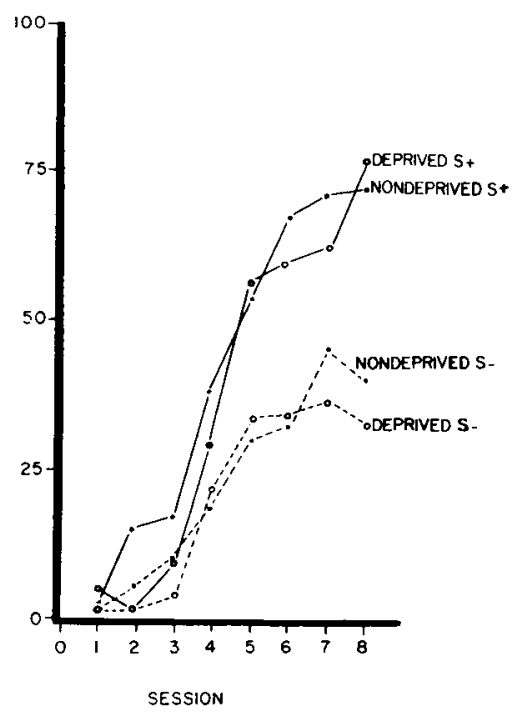

Fig. 1. Differential rabbit eyelid conditioning as a function of food deprivation level.

independent variables so the response protocols are presented separately for each variable. Figure 1 compares the differential conditioning performance to $S+$ and $S-$ for the deprived and nondeprived Ss. Food deprivation did not increase the rate of response acquisition. The trial of the fifth CR to S+ for the deprived group was 95.3 and for the nondeprived group was 90.4, $F(1,28)=.10$. The total number of CRs to S+ over the eight daily sessions was 61.3 for the food-deprived group and 66.5 for the nondeprived group, $F(1,28)=.20$.

The performance of the two different rabbit strains is depicted in Fig. 2. The American Dutch strain conditioned more rapidly than the New Zealand White (albine) strain. The trial of the fifth CR to S+ averaged 70.0 for the Dutch rabbits and 114.4 for the albinos, $F(1,28)=8.08$, $\mathrm{p}<.01, \omega^{2}=.186$. The total number of $\mathrm{CRs}$ to $\mathrm{S}+$ over all eight sessions averaged 84.3 for the Dutch rabbits and $\mathbf{4 4 . 7}$ for the albinos, $F(1,28)=11.68, p<.01$, $\omega^{2}=.256$.

Discrimination performance was assessed by a signal detection analysis of these data. Hit and false-alarm rates were estimated from response frequency to $S+$ and $S-$, respectively, after each $S$ had responded at a $50 \%$ or greater level to $\mathrm{S}+$ for one session. One albino and one Dutch rabbit were not included in this analysis because they failed to meet this criterion. Using the assumption that the sensory effects of signal ( $\mathrm{S}+$ ) and noise $(\mathrm{S}-$ ) can be described by measures that are distributed normally and have equal variances, $d^{\prime}$ and $\beta$ were estimated for each rabbit (Green \& Swets. 1966, Chap. 1-3). According to the theory of signal detection (TSD), $\mathrm{d}^{\prime}$ can be

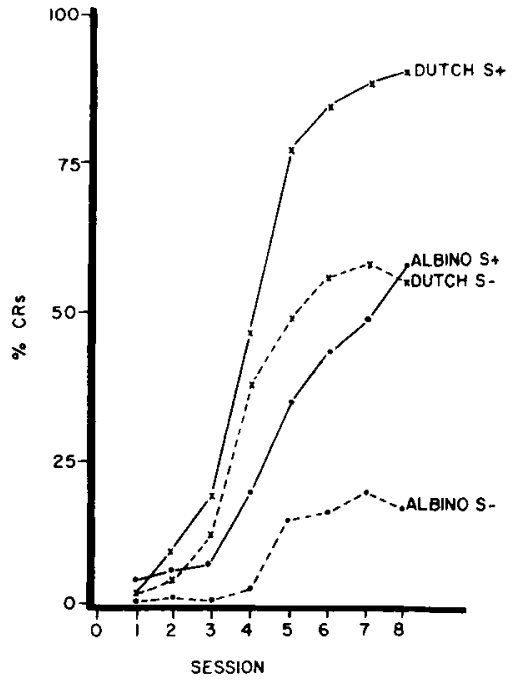

Fig. 2. Differential eyelid conditioning for New Zealand White and American Dutch rabbits.

interpreted as a sensitivity measure (discriminability) and $\beta$ as a measure of the general tendency to respond irrespective of which signal has been presented (responsivity).

This analysis indicated several interesting group differences. The average $d^{\prime}$ for the food-deprived rabbits was 1.80 and for the nondeprived rabbits was 1.19 , $F(1,26)=4.68, p<.05, \omega^{2}=.109$. The $\beta$ values for these two groups were not reliably different. The average $d^{\prime}$ for the albino rabbits was 1.86 and for the Dutch rabbits was $1.14, F(1,26)=5.93, p<.05$, $\omega^{2}=.141$. The average $\beta$ was 2.18 for the albinos and .59 for the Dutch. An analysis of $\log \beta$ indicated that the albino rabbits had a reliably more conservative criterion (i.e., larger $\beta$ ) than the Dutch animals, $\mathrm{F}(1,26)=9.87, \mathrm{p}<.01, \omega^{2}=.248$.

On the basis of the signal detection analysis, the albino rabbits seem to differ from the Dutch rabbit in two different ways. The albinos appear to be more sensitive and less responsive than the Dutch. Deprivation does not appear to increase responsivity, but does improve sensitivity.

\section{DISCUSSION}

The present results indicate that $18 \mathrm{~h}$ of food deprivation does not increase the rate of acquisition of a conditioned eyelid response in the rabbit. The food-deprivation manipulation also failed to increase the general responsivity of the animals as indicated by a signal detection $\beta$ measure. These findings are inconsistent with Hull's concept of general drive but are easily accounted for by Estes' theory of selective stimulus amplifiers.

The improvement in discrimination performance (i.e., enhanced $d^{\prime}$ ) is more consistent with Hull's position than with Estes's, but neither theory makes direct predictions about this measure. An alternative explanation for this improved discrimination performance might be to relate the effects of food deprivation to increased general arousal and therefore to better vigilance in the conditioning situation. The enhanced general arousal hypothesis emphasizes the improvement in sensory performance, while the Hullian general drive concept places more emphasis on the motor or output dimension of behavior.

Since the present investigation examined only a single, 18-h food-deprivation condition, one can plausibly argue that either fewer or more hours of food deprivation might show different effects. Rubin \& Brown (1969) reported that severe food deprivation (greater than $48 \mathrm{~h}$ ) was necessary to motivate Dutch rabbits to respond reliably in a food-motivated operant task. Consequently, it is possible that more severe deprivation conditions might have altered conditioning performance in the direction that Hullian drive theory predicts. However, the effectiveness of the 18-h deprivation in improving discrimination performance indicates that this manipulation is reasonably effective in altering some aspects of the rabbit's performance.

The analysis of the conditioning performance of two different rabbit strains in the present study provided an interesting comparative investigation. The results indicated that the American Dutch rabbit acquires the conditioned eyelid response more rapidly than the New Zealand White rabbit with the present conditioning procedure. The data also indicated that the Dutch animals are more responsive in the differential conditioning situation and, in signal detection terms, appear to hold a less conservative criterion (i.e., lower $\beta$ ) than the albino rabbits. This effect can be analogized to the Pavlovian descriptions of animals as "excitable" or "inhibited" types (Pavlov, 1928, p. 375), with the Dutch strain appearing to be less "inhibited" than the New Zealand White strain. In fact, the responsivity measure, $\beta$, might be a reasonable way to operationally define Pavlov's classifications. A "balanced" animal should show a score of approximately 1.0 (e.g., in the range of 0.5 to 2.0). "Excitable" types would have $\beta$ s of less than 0.5 , while "inhibited" types would have $\beta$ s greater than 2,0.

On the basis of the Dutch rabbits' performance in the present study, it seems reasonable to suggest that this $S$ population be considered seriously for future investigations. In addition to their good 
conditioning performance, the Dutch animals have several advantages over the albino rabbits in terms of handling and maintenance. Their smaller size makes them easier to handle and transport and permits the use of smaller housing cages. Since the Dutch rabbits eat and excrete less than the albino rabbits, the maintenance expense for the rabbit colony is reduced.

\section{REFERENCES}

ESTES, W. K. Outline of a theory of punishment In B. A. Campbell and R. M. Church (Eds.), Punishment and aversive behavior. New York Appleton-Century-Crofts, 1969. Pp. 57-83.

GREEN, D. M., \& SWETS, J. A. Signal detection theory and psychophysics New York: Wiley. 1966.

HULL, C. L. Principles of behavior. New York: Appleton-Century-Crofts, 1943.

MISANIN, J. R., \& CAMPBELL, B. A. Effects of hunger and thirst on sensitivity and reactivity to shock. Journal of Comparative \& Physiological Psychology, 1969, 69, 207-213. PAVLOV, I. P. (Translated and edited by W. H. Gantt.) Lectures on conditioned reflexes. New York: International Publishers, 1928.

RUBIN, H. B., \& BROWN, H. J. The rabbit as a subject in behavioral resarch. Journal of the Experimental Analysis of Behavior, 1969, 12, 663-667.

VAUGHAN, G. M., \& CORBALlis, M. C. Beyond tests of significance: Estimating strength of effects in selected Anova designs. Psychological Bulletin, 1969, 72, 204-213.

\section{Some preliminary observations on the behavior of Mongolian gerbils (Meriones unguiculatus) under seminatural conditions}

\author{
GORDON G. GALLUP, JR., and MARTIN S. WAITE \\ Tulane University, New Orleans, La. 70118
}

Small groups of Mongolian gerbils were released into an empty room so as to simulate free-ranging conditions and to study social patterns. While there was no tendency to maintain identifiable subgroups, the adult males positioned themselves into what appeared to be a dominance hierarchy. Dominant males initiated most of the fighting and were the only ones ever observed to mark objects in the room. In terms of sexual behavior, competition among males was completely absent, and multiple males were observed to copulate in sequence with any receptive female. Dominant males, however, were never observed to participate in sexual activities or to aggress against copulating subordinates. In general, males showed a much higher incidence of aggressive behavior and more footstomping than females. Footstomping episodes were most frequent during sexual activity and seemed to alert other members of the group.

Considerable interest has recently been shown in the Mongolian gerbil as a subject for behavioral research (e.g., Thiessen, 1968). However, a survey of the literature indicates that little is known about the free-ranging behavior of gerbils under naturalistic conditions. The work of Tanimoto (1943) is virtually the only published information on gerbils living outside the laboratory or caged colony situation, but unfortunately provides very

\section{few behavioral details.}

It is widely felt that information concerning natural behavior patterns is vital in directing research priorities and for achieving accurate psychological perspectives of any animal. The present study represents the preliminary results of an ongoing attempt to establish a biologically relevant set of behavioral observations that may help provide a more informed basis for subsequent laboratory work on this species. There are, however, several problems associated with such an objective. It is likely that the current research population of gerbils in this country was subject to an immigration sampling effect (Klopfer \& Hailman, 1967). Virtually all available gerbils have been laboratory bred since 1935 (Rich, 1968) from a small number of original animals, which may not have been representative of the Mongolian population. Moreover, approximations to the natural habitat of gerbils are difficult to achieve due to differences in available food, climate, terrain, etc. Also, since gerbils are burrowing animals (Allen, 1940) provisions for burrowing would make continuous observation without intervention difficult. The conditions that prevailed in the present study were in no way meant to provide for a perfect approximation, but, rather, represent an attempt to assess behavioral capacities under free-ranging conditions.

\section{SUBJECTS}

The Ss were eight male and seven female adult Mongolian gerbils (Meriones unguiculatus), which prior to the experiment had been reared from birth in small groups of littermates containing both sexes. Group size ranged from two to five animals. All Ss were experimentally naive and had been subjected to only minimal handling before participating in the study. APPARATUS

An empty $9 \times 13 \mathrm{ft}$ room .was selected for making behavioral observations. The wooden floor of the room was covered with cedar shavings and an observation stool was situated near one wall. Natural light was provided through a window with a photoperiod of about $10 \mathrm{~h}$ per day and temperature varied over days from approximately $60^{\circ}$ to $85^{\circ} \mathrm{F}$.

Two water bottles were affixed to the wall under the window and food in the form of Purina Rat Chow and sunflower seeds was periodically scattered on the floor in the middle of the room. Paper was also made available for shredding.

$$
\text { PROCEDURE }
$$

For purposes of identification, each animal had small amounts of fur shaved from various portions of its body and head, and Ss were assigned numbers on the basis of these markings. All 15 animals were then permanently released into the room and were allowed 2 weeks to adapt to the new environment. During the adaptation period, informal observations were made every day in order to perfect recording techniques and to determine what kinds of behaviors warranted study.

After the 2-week adaptation, all animals were systematically observed for one or two $30-\mathrm{min}$ periods each day, with one 